\title{
Editorial
}

\section{Progress in Postvention}

\section{A Call to a Focused Future to Support Those Exposed to Suicide}

\author{
Myfanwy Maple ${ }^{1}$, Vita Poštuvan², and Sharon McDonnell ${ }^{3,4}$ \\ 'School of Health, University of New England, Armidale, NSW, Australia \\ ${ }^{2}$ Slovene Centre for Suicide Research, Andrej Marušič Institute, University of Primorska, Slovenia \\ ${ }^{3}$ Suicide Bereavement UK, Manchester, UK \\ ${ }^{4}$ Centre for Mental Health and Safety, University of Manchester, UK
}
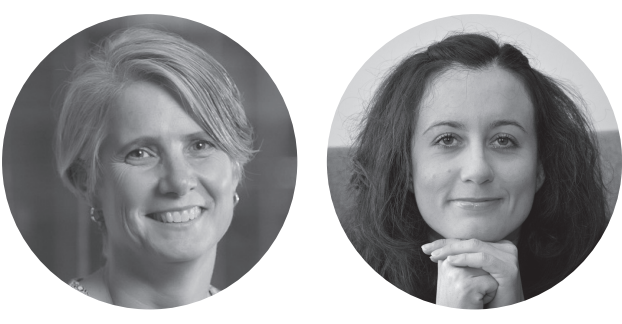

In the late 1960s, Edwin Shneidman first focused attention on the needs of those bereaved by suicide and coined the term postvention. To quote him directly from the original 1972 source:

\begin{abstract}
We hear a great deal about suicide prevention and interventions. A benign community ought routinely to provide postventive mental health care for the survivor-victims of suicidal deaths. Postvention is prevention for the next decade and for the next generation. Of the three possible (temporal) approaches to mental health crises - prevention, intervention and postvention - in the case of suicide at least, postvention probably represents the largest problem and thus presents the greatest area for potential aid. If there are about 50,000 committed [sic] suicides in the United States every year - not counting the couple million "subintentioned" presently labelled natural, accidental, and homicidal deaths - then there are at least 200,000 survivor-victims created each year whose lives are ever after benighted by that event. (p. x)
\end{abstract}

Reports discussing how Shneidman quantified those affected by suicide generally suggest that the estimate of so-called survivors was not based on any empirical data, but rather on approximations used to gauge the number of people affected by catastrophic events that occurred around the same period (cf. Berman, 2011). Many in the suicide prevention field have cited Shneidman (including the authors of this editorial) and most have got his original figure wrong, claiming that it was six. It is clear from the original source that this is mathematically incorrect; the number originally proposed never equaled six survivors, but instead four $(200,000 / 50,000=4)$. However, Shneidman did later claim there are an "estimated half-dozen survivor-victims whose lives are thereafter benighted by that event" (Shneidman, 1973, p. 22).

The scope of those affected by suicide has been the focus in the ensuing decades. Researchers have tried to un- derstand who is exposed to and affected by suicide, in what ways those exposed to suicide deaths are affected (along a continuum from a small effect to lifelong bereavement), and how this is similar to or different from responses to other deaths, most commonly unexpected and/or violent deaths. Yet, over the 50-year period since Shneidman first initiated the focus on those affected by suicide deaths, only 443 articles have been published on suicide bereavement according to a recent systematic review (Maple et al., 2018). This review did demonstrate a continued upward trajectory of published research over time, however, and indicated that this research primarily investigated exposure to suicide, attitudes to suicide deaths, and comparisons in responses to suicide deaths and other deaths.

At the same time as this review was being undertaken, a survey of those actively involved in suicide exposure research was also carried out (Andriessen, Castelli Dransart, Cerel, \& Maple, 2017). The outcome of these two pieces of work, undertaken by a taskforce of the International Association of Suicide Prevention's Special Interest Group on Suicide Bereavement and Postvention, was three broad recommendations: (1) that there is a need to fully understand the rate of exposure beyond immediate kinship and across minority groups and different cultures, (2) that theory-driven research is required, and (3) that evidence-based interventions should be sought and strengthened through collaboration with service providers and people with lived experience.

Significant leaps forward have been made to respond to these recommendations. The first is adequately defining the population of those affected by suicide. This has now been done in several continents, including through the use of the United States-based General Social Survey by Feigelman, Cerel, McIntosh, Brent, and Gutin (2018). Large community-based studies have also been conducted 
in Australia (Maple \& Sanford, 2019) and the United Kingdom (McDonnell, Hunt, Flynn, Smith, \& McGale, 2018), and there is one currently underway in Canada (Sanford, personal communication, May 2019) and possibly others elsewhere. These studies are demonstrating that the percentage of a population that have been bereaved by, or exposed to, suicide is greater than $50 \%$. Such findings indeed indicate there are not six (c.f. Cerel et al., 2018) exposed to suicide, but rather this experience is common. Cerel and colleagues (2019) utilized data from one US state to determine the number of people exposed in that location to each suicide death, calculating that for each suicide death 135 people are exposed. In addition to determining how common exposure to suicide is, these contemporary reports are also showing that multiple exposures to suicide (both death and attempts) are also common, and that there appears to be a cumulative effect whereby the likelihood of adverse outcomes is increased with each exposure. This adds further nuance to studies investigating diverse characteristics that influence poorer outcomes following suicide exposure retrieved in recent systematic reviews examining exposure among kin (Pitman, Osborn, King, \& Erlangsen, 2014) and non-kin (Maple, Cerel, Sanford, Pearce, \& Jordan, 2017). Thus, we can now confidently say that Shneidman's initial estimates of four (or six) suicide survivors/those bereaved is a significant underestimation.

Now that this work has been done, and we can say with more confidence that exposure to suicide is widespread, attention can be given to the important questions that remain, namely, theoretical and epidemiological research across a range of settings that in turn can provide evidence to inform interventions to support those affected by suicide. To achieve this task, and following the example of Silverman, Berman, Sanddal, O'Carroll, and Joiner (2007) in relation to suicide more broadly, it is timely that agreed nomenclature be determined. The current terminology used is problematic as it is both clumsy and often inaccurate in describing a broad range of experiences. Earlier work on suicide bereavement and postvention frequently used the term survivor-victims, which stems directly from Shneidman's original thoughts. This has more recently been replaced by bereaved by suicide, survivors of suicide, and suicide exposed. Yet this contemporary terminology remains somewhat ambiguous, since terms such as suicide survivors can refer both to those who have been bereaved by suicide and those who have attempted suicide. This frustrates efforts to extend understanding about the impact of suicide. For example, in the previously mentioned review (Maple et al., 2018), upwards of $98 \%$ of records retrieved in the systematic search were assessed as irrelevant to suicide exposure during screening. This was caused by a lack of consistent keywords and terminology used to identify those exposed to or affected by suicide.
The recently proposed Continuum of Survivorship (Cerel, McIntosh, Neimeyer, Maple, \& Marshall, 2014) uses terms associated with survivors of suicide, which are more common in the United States (understandably as this is where the majority of the authors of this model reside). While this may not be preferred terminology globally, this model does provide initial theorizing about the range of responses that may be experienced across different relationship types. This continuum is based on an attachment model suggesting that those with closer attachments to the deceased will experience the most significant and long-lasting impact from the suicide. Further, the model suggests that those most affected by suicide will be bereaved by that event, through either short- or long-term bereavement. The relationships proposed to fit within these groups are close or kinship relationships, including but not limited to immediate and extended family and close friends. Bereavement in and of itself requires grieving the loss of an emotional attachment. Yet, the variety of experiences reported in the aforementioned community surveys go well beyond attached relationships. Thus, while noting the limitation of the attachment model and assumed bereaved upon which it is based, the continuum model now requires rigorous testing to understand for whom, and in which situations, is such a model relevant and/or in what ways does it require expansion.

Recent reports, such as those from the national surveys mentioned above, make it clear that any model must be inclusive of a range of experiences with the flexibility to take into consideration those seemingly peripherally exposed to a suicide death through to those whose lives are changed forever. A broad model will need to acknowledge the experiences of "zero responders" (Burns, Douglas, \& Hu, 2019), that is, the community members who find the deceased, but who may never have known the person. It will also need to incorporate the experiences of first responders, such as ambulance officers, police, and firefighters who may experience cumulative effects of multiple exposures to suicide deaths, which are well beyond the general community, for example, the 30 exposures on average among police reported by Cerel and colleagues (2018). These individuals may have never known or had a connection to the person who has died that they have professionally responded to, yet their repeated exposure may heighten their own suicide risk (c.f. Kimbrel et al., 2016), adding to the evidence that individuals in these professions have higher levels of suicidal thoughts and behaviors than others (Stanley, Hom, \& Joiner, 2016). We also need to consider second responders, or health and social care service staff who may have established a relationship through therapeutic engagement in a workplace context, but again may not necessarily have an attached emotional connection. These individuals may also experience one, or more commonly, many exposures 
to suicide in their professional lives often without the emotionally attached connection that underlies the proposed continuum of exposure. These occupation groups - particularly for female health professionals (Milner, Maheen, Bismark, \& Spittal, 2016) - are at heightened risk of suicide, and so they may further be exposed through deaths of peers as well as family connections.

For these reasons, further theorizing is needed to extend the proposed continuum to include nonattached individuals, and those with relationships that extend beyond the most visible ones, as well the role of cumulative exposure to suicide. Additional epidemiological studies are required to determine how this exposure is experienced within minority groups (e.g., Indigenous populations, LGBTIQ+ people, and close-knit, prison and rural communities). Further qualitative work is required to understand how people make meaning of these events and how this relates to their identification with the suicide death and the effect this identification has. For example, in a qualitative project reported by Bartik, Maple, and McKay (2015), rural young people talked about how they did not feel justified in feeling as bad as they did following the loss of a friend to suicide, rather believing that where support services were available these should be directed to immediate family and friends. Yet, while these young people discussed being disenfranchised in their grieving (c.f. Doka, 2002), they were simultaneously exhibiting extreme risk behaviors, and were found to consider suicide in normalizing and glorified ways.

This example, along with many other published in the suicide exposure and bereavement literature, shows that beyond the proposed need for agreed terminology and epidemiological and theoretical work, innovative development is urgently required to understand which interventions might work to reduce adverse outcomes associated with exposure across diverse communities and population groups. To date, intervention studies in this area are almost completely absent from the literature. Robust evaluation of the acceptability and efficacy of different interventions across different population groups remains a priority. Where postvention practices are being undertaken in some areas across the globe, both formally (some proactive and some reactive) and informally through peer supports (such as well-established peer and professionally led grief support groups), evaluations should be undertaken and published. Ideally, these evaluations should draw on longitudinal outcome data. Supporting supporters - those who work professionally in the field - is also crucial (e.g., McDonnell et al., 2015). For those working in the field, there are questions about where and when secondary trauma intersects with meaningful and purposeful occupation. Looking outside suicidology to areas such as critical incident training may enable established evidence-based training to be adapted to the specific needs of those exposed to suicide (e.g., Everly \& Mitchell, 1997). These advances could inform the development of quality standards that others could then utilize to develop training for supporters that is targeted to specific populations.

While acknowledging these adverse outcomes and the need for evidence informed support, focus is also required on the emerging field of posttraumatic growth following exposure to suicide (c.f. Levi-Belz, 2019; Moore, Cerel, \& Jobes, 2015). Those who find new meaning and purpose in life following another's suicide death have much to offer to inform the development of appropriate and timely interventions to support those who struggle. They can help answer questions about the characteristics that enable some people to grow and thrive following these exposures, and the ways in which these characteristics might be harnessed to inform interventions to support others who need assistance. It is incumbent upon us as suicidologists to ensure that the messages of harm and risk are tempered with stories of survival. Just as the media are encouraged to moderate their reporting of suicide and include stories of those who have been in a suicidal crisis and recovered, so too should we be looking at the ways in which people can thrive following exposure to suicide.

While we have not forgotten to cite Shneidman over the past 50 years, the crucial part of his message, that we "ought routinely provide postventive mental health care" for those exposed is yet to be realized. His key message that "postvention probably represents the largest problem and thus presents the greatest area for potential aid" is undisputed. We are now aware of the magnitude of the problem, the level of unmet need, and the urgency to address it. Thus, our challenge now is to strengthen the "postvention pillar" of suicide prevention originally proposed by Shneidman. Current research enables us to confidently claim that exposure to suicide is widespread. The urgency to address this phenomenon, to help influence policy and practice in order to save lives cannot be underestimated.

\section{References}

Andriessen, K., Castelli Dransart, D., Cerel, J., \& Maple, M. (2017). Current postvention research and priorities for the future: Results of a survey. Crisis, 38(3), 202-206. https://doi. org/10.1027/0227-5910/a000459

Bartik, W., Maple, M., \& McKay, K. (2015). Suicide bereavement and stigma for young people in rural Australia: A mixed methods study. Advances in Mental Health, 13(1), 84-95. https://doi.org/ 10.1080/18374905.2015.1026301

Berman, A. (2011). Estimating the population of survivors of suicide:Seeking an evidence base. Suicide and Life-Threatening Behavior, 410(1), 110-116. https://doi.org/10.1111/j.1943-278X. 2010.00009.x 
Burns, P., Douglas, K., \& Hu, W. (2019). Primary care in disasters: Opportunity to address a hidden burden of health care. Medical Journal of Australia, 210(7), 297-299. https://doi.org/10.5694/ mja2.50067

Cerel, J., Brown, M., Maple, M., Singleton, M., van de Venne, J., Moore, M., \& Flaherty, C. (2019). How many people exposed to suicide? Not Six. Suicide and Life-Threatening Behavior, 49(2) 529-534. https://doi.org/10.1111/sltb.12450

Cerel, J., Jones, B., Brown, M., Weisenhorn, D., \& Patel, K., (2018). Suicide exposure in law enforcement officers. Suicide and Life-Threatening Behavior. Advance online publication. https:// doi.org/10.1111/sltb.12516

Cerel, J., Mclntosh, J., Neimeyer, R., Maple, M., \& Marshall, D. (2014). The continuum of "survivorship": Definitional issues in the aftermath of suicide. Suicide and Life-Threatening Behavior, 44(6), 591-600. https://doi.org/10.1111/sltb.12093

Doka, K. (Ed.). (2002). Disenfranchised grief. Champaign, IL: Research Press.

Everly, G., Jr., \& Mitchell, J. T. (1997). Critical incident stress management (CISM): A new era and standard of care in crisis intervention. Ellicott City, MD: Chevron.

Feigelman, W., Cerel, J., Mclntosh, J., Brent, D., \& Gutin, N. (2018). Suicide exposures and bereavement among American adults: Evidence from the 2016 General Social Survey. Journal of Affective Disorders, 227, 1-6. https://doi.org/10.1016/j. jad.2017.09.056

Kimbrel, N., Pennington, M., Cammarata, C., Leto, F., O'stiguy, W., Gulliver, S., \& Carroll, P. (2016). Is cumulative exposure to suicide attempts and deaths a risk factor for suicidal behavior in firefighters? A preliminary study. Suicide and Life-Threatening Behavior, 46(6), 669-677. https://doi.org/ 10.1111/sltb.12248

Levi-Belz, Y. (2019). With a little help from my friends: A follow-up study on the contribution of interpersonal characteristics to posttraumatic growth among suicide-loss survivors. Psychological Trauma: Theory, Research, Practice, and Policy. Advance online publication.

Maple, M., Cerel, J., Sanford, R., Pearce, T., \& Jordan, J. (2017). Is exposure to suicide beyond kin associated with risk for suicidal behavior? A systematic review of the evidence. Suicide and Life-Threatening Behavior, 47(4), 461-474. https://doi. org/10.1111/sltb.12308

Maple, M., Pearce, T., Sanford, R., Cerel, J., Castelli Dransart, D., \& Andriessen, K. (2018). A systematic mapping of suicide bereavement and postvention research and a proposed strategic research agenda. Crisis, 39, 275-282. https://doi. org/10.1027/0227-5910/a000498

Maple, M., \& Sanford, R. (2019). Suicide exposure and impact within a non-representative Australian community sample. Death Studies. Advance online publication. https://doi.org/10.1080/0 7481187.2018.1554609

McDonnell, S., Chew-Graham, C., Kapur, N., McGale, B., Smith, S., Shaw, J., \& Cordingley, L. (2015). Postvention assisting those bereaved by suicide: Parental suicide bereavement manual for health care practitioners. Manchester, UK: University of Manchester.

McDonnell, S., Hunt, I. M., Flynn, S., Smith, S., \& McGale B. (2018). Impact of Suicide in the UK Survey. Manchester, UK: University of Manchester.
Milner, A., Maheen, H., Bismark, M., \& Spittal, M. (2016). Suicide by health professionals: A retrospective mortality study in Australia, 2001-2012. Medical Journal of Australia, 205(6), 260-265. https://doi.org/10.5694/mja15.01044

Moore, M., Cerel, J., \& Jobes, D. (2015). Fruits of trauma? Posttraumatic growth among suicide-bereaved parents. Crisis, 36, 241248. https://doi.org/10.1027/0227-5910/a000318

Pitman, A., Osborn, D., King, M., \& Erlangsen, A. (2014). Effects of suicide bereavement on mental health and suicide risk. The Lancet Psychiatry, 1(1), 86-94. https://doi.org/10.1016/S22150366(14)70224-X

Shneidman, E. (1972). Foreword. In A. Cain (Ed.), Survivors of suicide (pp. ix-xi). Springfield, IL: Charles C. Thomas Publishers.

Shneidman, E. (1973). On the nature of suicide. San Francisco, CA: Jossey-Bass.

Silverman, M., Berman, A., Sanddal, N., O'Carroll, P., \& Joiner, T. (2007). Rebuilding the Tower of Babel: A revised nomenclature for the study of suicide and suicidal behaviors part 2: Suicide-related ideations, communications, and behaviors. Suicide and Life-Threatening Behavior, 37(3), 264-277. https://doi. org/10.1521/suli.2007.37.3.264

Stanley, I., Hom, M., \& Joiner, T. (2016). A systematic review of suicidal thoughts and behaviors among police officers, firefighters, EMTs, and paramedics. Clinical Psychology Review, 44, 25-44. https://doi.org/10.1016/j.cpr.2015.12.002

Published online November 15, 2019

Myfanwy Maple, PhD, is Professor of Social Work at the University of New England, Australia. Dr. Maple's research focuses on trauma and loss, with a particular emphasis on understanding risk and resilience following exposure to suicide. She currently serves as a co-chair of the IASP special interest group on suicide bereavement and postvention.

Vita Poštuvan, PhD, is Deputy Head of the Slovene Centre for Suicide Research (UPIAM), and involved in research, therapeutic and public-health work related to suicidal behavior, bereavement, and crisis interventions. Dr. Poštuvan currently serves as a co-chair of the IASP special interest group on suicide bereavement and postvention.

Sharon McDonnell, PhD, is Managing Director of Suicide Bereavement UK. Her team have translated research findings into PA BBS evidence-based suicide bereavement training. She is currently leading a national suicide bereavement survey in the UK. Dr. McDonnell currently serves as a co-chair of the IASP special interest group on suicide bereavement and postvention.

\section{Myfanwy Maple}

School of Health

University of New England

Armidale NSW 2351

Australia

mmaple2@une.edu.au 